\title{
Avaliação da ativação muscular do centro de força de mulheres nuligestas
}

\author{
Evaluation of muscular activation of center of force \\ for women nulliparous
}

\author{
Letícia Fernandez Frigo', Melissa Medeiros Braz² \\ 'Centro Universitário Franciscano (Unifra), Santa Maria, RS, Brasil. \\ Universidade Federal de Santa Maria (UFSM), Santa Maria, RS, Brasil.
}

Recebido em: 17/11/2015 / Aceito em: 16/05/2016

leticia_frigo@yahoo.com.br

\section{RESUMO}

O centro de força é composto por um conjunto muscular responsável pela estabilização do complexo lombo-pélvico. Objetivo: avaliar a musculatura responsável pela estabilização lombo-pélvica em nuligestas. Método: este estudo contou com a participação de 40 mulheres, com idade média de 23,03 $\pm 3,43$ anos. Foi realizada uma avaliação da ativação da musculatura profunda do abdome, musculatura do tronco, perineal e respiratória. Os resultados foram analisados estatisticamente por meio dos valores de frequência absoluta e relativa. Resultados: $55 \%$ das avaliadas possuem ativação ineficiente da musculatura profunda do abdome, $65 \%$ apresentaram recrutamento normal do multífido, $75 \%$ das avaliadas obtiveram valores fracos para o teste de abaixar a perna estendida, 77,2\% das mulheres têm uma força perineal satisfatória. A avaliação da Pimáx entre as avaliadas foi $60 \%$ satisfatória e $40 \%$ insatisfatória e Pemáx foi $57,5 \%$ satisfatória e $42,5 \%$ insatisfatória. Considerações finais: as avaliadas mostraram maior dificuldade na ativação dos músculos profundos do abdome, sendo que os resultados na avaliação dos demais grupos musculares mostraram-se satisfatórios.

Palavras-chave: Fisioterapia; Mulher; Coluna.

\section{ABSTRACT}

The center of force or core is composed of mus cles responsible for stabilizing the lumbar-pelvic complex. Objective: to evaluate the muscles responsible for lumbar-pelvic stability in nulliparous women. Method: this study had the participation of 40 women, mean age of $23.03 \pm 3.43$. It was made an evaluation of the following muscles activation: abdomen deep muscles, trunk, perineal and breathing muscles. The results were analyzed statistically by means of absolute and relative frequency values. Results: $55 \%$ of the assessed women have inefficient activation of abdomen deep muscles, $65 \%$ showed normal recruitment of the multifidus, $75 \%$ of subjects obtained weak results for the test of lowing the extended leg, $77.2 \%$ of women have a satisfactory perineal strength. The Pimax evaluation was classified in $60 \%$ satisfactory and $40 \%$ unsatisfactory and Pemax was classified in $57.5 \%$ satisfactory and $42.5 \%$ unsatisfactory. Closing remarks: volunteers showed greater difficulty in activating abdomen deep muscles. Evaluation results of other muscle groups were satisfactory.

Keywords: Physiotherapy; Woman; Spine.

\section{INTRODUÇÃO}

A região lombo-pélvica constitui o centro de atividade do corpo humano, onde se localiza o centro de gravidade. É uma cinta muscular responsável pela estabilização e transferência de peso da coluna e do tronco, reduzindo as sobrecargas, durante os movimentos da cadeia cinemática. Contribui para sustentação estática do peso, biomecânica normal e postura. ${ }^{1-3}$

Os músculos do tronco, além de agirem como motores primários ou antagonistas do movimento, servem como protetores da coluna, promovendo base estável para que os músculos dos membros executem sua função. O recrutamento inadequado dessa musculatura gera sobrecarga excessiva sobre todos os componentes da coluna vertebral. ${ }^{4}$ 
Mulheres nuligestas tendem a apresentar alterações funcionais relacionadas a posturas inadequadas, porém ainda não sofreram alterações biomecânicas oriundas da gestação, momento no qual estas acabam tendo sobrecargas em determinados grupos musculares. Ainda, de acordo com os estudos de Silva et al., ${ }^{5}$ realizado na cidade de Pelotas, com 3.182 indivíduos, sendo $56,8 \%$ mulheres e Matos et al., ${ }^{6}$ realizado com 775 indivíduos, as mulheres são mais vulneráveis a alterações músculo-esqueléticas.

As mulheres apresentam maior queixa de dores musculares, possivelmente em decorrência de algumas características anátomo-funcionais, tais como menor massa óssea, menor resistência muscular e articulações mais instáveis e, em função de sua força muscular ser $30 \%$ menor do que a dos homens, em média. ${ }^{6}$

A estabilidade lombo-pélvica é definida como a capacidade de controlar o movimento da coluna lombar e da pelve em relação a uma posição neutra, apresentando grande importância nas reabilitações das enfermidades da coluna. Um centro de força estável tem a capacidade de transferir cargas de uma forma eficiente para as articulações e realizar uma transmissão de força eficaz. ${ }^{7}$

A manutenção da estabilidade da coluna requer uma coordenação dos elementos que atuam no movimento. A falta de estabilidade produz uma exacerbação dos movimentos da coluna, quando esta é submetida a determinadas forças. A instabilidade gera movimentos ineficientes e pode levar à ocorrência de lesões. ${ }^{8}$ Isto destaca a importância do treinamento da musculatura estabilizadora para a manutenção da estabilidade da coluna. $^{9}$

Panjabi ${ }^{10}$ propôs um modelo de interação entre os componentes que fornecem estabilidade. São descritos três sistemas: as vértebras, discos e ligamentos constituem o sistema passivo; o sistema ativo é composto pelos músculos e tendões, em torno da coluna vertebral; os nervos e o sistema nervoso central formam o controle, que recebe e capta as alterações de equilíbrio e determina os ajustes específicos por meio da musculatura da coluna, restaurando a estabilidade.

Quando cargas adicionais ou movimentos bruscos são aplicados ao corpo o controle neural pode alterar a estratégia de recrutamento muscular com o objetivo de reforçar temporariamente a estabilidade da coluna, além das exigências normais. A disfunção de qualquer um dos subsistemas gera falha na estabilização, diminuição de potência, controle neuromuscular e resistência muscular. ${ }^{10,11}$

Embora a função de cada sistema possa ser atenuada por lesões ou desuso, esta pode ser restabelecida pelo recrutamento do sistema ativo. Os músculos têm importante função na estabilização, já que a coluna é altamente instável quando é submetida a cargas; daí a importância da atividade muscular. Os grandes músculos são responsáveis pela produção e controle do movimento da coluna. A musculatura mais profunda atua com finalidade segmentar. ${ }^{7,8}$

O multífido atua diretamente como estabilizador e protetor da coluna e da pelve, agindo em ação preparatória antes da movimentação dos membros. O transverso do abdome e o multífido são os únicos músculos ativos, durante todos os movimentos do tronco. ${ }^{1,2,8}$
As fibras do transverso do abdome correm horizontalmente em torno do abdome, formando um cilindro rígido. Essa tensão lateral pode resultar em limitação dos movimentos e aumento da estabilidade; além disso, o aumento da pressão intra-abdominal anterior à coluna gera força contra a lordose lombar, o que pode aumentar a estabilidade da coluna, em diversas posturas. ${ }^{4}$

O diafragma funciona como teto do centro. A estabilidade é transmitida à coluna por contração do diafragma e aumento da pressão intra-abdominal. Os músculos do assoalho pélvico também são totalmente ativados na estabilização e recrutam-se também com a contração do transverso do abdome. O assoalho pélvico é responsável pelo suporte dos órgãos pélvicos e conteúdo abdominal, especialmente quando em pé, ao exercer força durante o movimento. ${ }^{12,13}$

Destaca-se a necessidade do bom funcionamento da musculatura global em torno do centro. É fundamental a coordenação de toda a musculatura profunda da coluna para que se garanta uma movimentação harmônica, estabilidade e bom desempenho funcional. ${ }^{12,13}$

esta forma, é necessário um enfoque que considere a importância do trabalho integrado, em que toda a cadeia cinética opere sinergicamente para gerar força, reduzir sobrecargas e possibilitar a estabilização dinâmica contra forças anormais..$^{1,14}$

Diante do exposto, este trabalho teve como objetivo avaliar a ativação muscular do centro de força de mulheres nuligestas.

\section{MÉTODO}

Esta pesquisa se caracteriza por possuir abordagem quantitativa do tipo descritiva. A amostra deste estudo foi composta por mulheres em idade fértil, nuligestas, que faziam uso de anticoncepcional, com faixa etária de 20 a 35 anos.

A amostra foi constituída de 40 mulheres na menacme, selecionadas conforme os critérios de exclusão que seguem: mulheres com patologias degenerativas da coluna, submetidas a cirurgias ortopédicas ou abdominais, com comprometimentos cognitivos e não alfabetizadas. Esta pesquisa foi aprovada previamente pelo Comitê de Ética e Pesquisa, do Centro Universitário Franciscano, sob o número de registro 162.2009.2. A coleta de dados foi realizada individualmente na clínica de Fisioterapia da referida instituição, em horário e local previamente agendados pelas pesquisadoras.

Para identificar as participantes e coletar dados relevantes à pesquisa utilizou-se um questionário de anamnese desenvolvido pelos pesquisadores, contendo questões fechadas sobre hábitos e informações das participantes como prática de exercícios físico, profissão e hábitos como o tabagismo, patologias respiratórias referidas, constipação intestinal e problemas de visão.

Para avaliar a ativação da musculatura profunda do abdome, foi utilizado o esfigmomanômetro como unidade de biofeedback pressórico. Posicionada em supino, primeiramente a avaliada foi ensinada a ativar a musculatura profunda abdominal com instruções verbais, como "encolher o abdome levando o umbigo em direção à coluna no tempo expiratório". ${ }^{3}$ A investigada assumiu a posição neutra da coluna e tentava mantê-la, 
enquanto encolhia suavemente e deprimia os músculos abdominais. Para iniciar o teste, o sujeito assumiu a posição prona sobre a unidade de biofeedback de pressão. O esfigmomanômetro era nivelado ao centro da parede abdominal. O manômetro era insuflado até $70 \mathrm{mmHg}$. Então a avaliada foi instruída a "puxar para dentro o abdome", para ter uma ativação total da musculatura abdominal, incluindo transverso do abdome. O resultado esperado foi que, após a contração, a pressão caísse de 4 a $10 \mathrm{mmHg}$ e que fosse mantida por 10 segundos. $^{3}$ Neste caso, a ativação era considerada satisfatória. Caso a diferença de pressão gerada ou o tempo não estivesse dentro destes parâmetros, a ativação era considerada insatisfatória. ${ }^{3}$ Neste teste, foi registrada a variação da pressão gerada pela contração, bem como o tempo de manutenção.

A estabilidade da musculatura espinhal foi avaliada mediante o teste de abaixamento das pernas estendidas. ${ }^{15} \mathrm{~A}$ voluntária foi colocada na posição supina. $\mathrm{O}$ esfigmomanômetro foi colocado sob a coluna vertebral lombar, aproximadamente à altura de L4-L5. O manômetro foi insuflado até $40 \mathrm{mmHg}$. Os joelhos da avaliada foram mantidos em completa extensão, enquanto os quadris foram flexionados ativamente até 90 graus. A avaliada foi instruída a repetir a manobra de contrair o abdome e, em seguida, achatar ao máximo possível as costas sobre a maca e o esfigmomanômetro. A avaliada foi instruída a baixar as pernas em direção à maca, ao mesmo tempo em que mantinha as costas retas e a pelve neutra. $O$ teste terminou quando a pressão insuflada começou a diminuir. Então foi medido o ângulo coxo-femural no qual ocorreu a alteração da pressão do manguito com um goniômetro, sendo utilizado o seguinte escore: normal: capaz de alcançar de 0 a $15^{\circ}$, a partir da maca; bom: capaz de alcançar 16 a $45^{\circ}$ a partir da maca; regular: capaz de alcançar 46 a $75^{\circ}$ a partir da maca; fraco: capaz de alcançar 75 a $90^{\circ}$ a partir da maca; e traço: quando a avaliada é incapaz de manter a pelve neutra. ${ }^{15}$

Para verificar a força da musculatura perineal foi utilizado o aparelho Perina. Inicialmente, a avaliada foi colocada em posição ginecológica, com o assoalho pélvico e membros inferiores desnudos. Esta foi orientada a não realizar manobra de valsalva e evitar a substituição da contração da musculatura perineal pelos adutores ou glúteos. Para avaliar a contração isolada do assoalho pélvico, primeiramente a voluntária foi informada sobre o que se esperava dela. Foram dados comandos verbais como "impeça a passagem de urina" até que foi observada a contração da musculatura perineal. As voluntárias foram instruídas a realizar uma contração perineal no tempo expiratório da respiração. A haste de látex do aparelho, previamente esterilizada com álcool etílico $70 \%$ e revestida por um preservativo descartável, foi introduzida na vagina.

O nível de pressão na escala numérica foi zerado, e foi solicitado à mulher uma contração perineal com o máximo de esforço. Foi realizada uma sequência de 5 contrações com intervalo de um minuto entre cada uma. $\mathrm{O}$ resultado foi constituído pelo maior valor numérico alcançado de 5 contrações. Essa medida repercute a capacidade de recrutamento das fibras da musculatura do assoalho pélvico e o fechamento do esfíncter ure- tral. Os valores normais de força perineal em decúbito dorsal situam-se entre 20 e $60 \mathrm{mmHg} \cdot{ }^{16-19}$ Se a pressão gerada se situasse entre estes valores, a ativação da musculatura perineal era considerada satisfatória, caso os valores estivessem abaixo do esperado, a ativação era considerada insatisfatória.

Para avaliar a capacidade do multífido de estabilizar o tronco durante movimentos dinâmicos das extremidades as avaliadas foram colocadas na posição de quatro apoios com a pelve em posição neutra, utilizando o controle muscular para permanecer nesta posição. Foram solicitadas então que realizassem as seguintes manobras: fletir um membro superior e mantê-lo nesta posição, posteriormente elevar o membro inferior estendido e mantê-lo, e por fim associar os dois movimentos de forma contralateral, sendo classificadas com o escore a seguir: normal: capaz de realizar a elevação da perna e do braço contralateral, em ambos os lados, enquanto mantém a pelve neutra e manter por 20 a 30 segundos; bom: capaz de manter a pelve neutra, enquanto realiza a elevação simples da perna e manter por 15 a 20 segundos; regular: capaz de realizar a elevação simples do braço, enquanto mantém a pelve neutra e manter por 15 a 20 segundos; fraco: incapaz de manter a pelve neutra, enquanto realiza a elevação simples do braço; traço: incapaz de elevar o braço ou a perna da maca até a posição estendida. ${ }^{15}$

A força dos músculos respiratórios foi avaliada com um manovacuômetro da marca Comercial Médica. Este disponibiliza as medidas de pressão inspiratória máxima (PImáx), que corresponde ao índice da força diafragmática; e a pressão expiratória máxima (PEmáx), que mede a força da musculatura abdominal e intercostal. As medidas das pressões foram realizadas com os indivíduos sentados e com as narinas ocluídas. Para a medida da PImáx, as avaliadas expiraram até o volume residual e, posteriormente, geraram um esforço inspiratório máximo no bocal. Para a medida de PEmáx foi realizada uma inspiração até a capacidade pulmonar total e, em seguida, um esforço máximo expiratório foi gerado contra o bocal. Os valores ideais para cada avaliada foram calculados, individualmente, de acordo com a equação: PImáx: $y=-0,49$ (idade) $+110,4$, com erro-padrão da estimativa $=9,1$. PEmáx: $y=-0,61$ (idade) $+115,6$; com erro-padrão da estimativa $=11,2 \cdot{ }^{20,21}$ Os dados foram analisados por frequência e percentual.

\section{RESULTADOS}

Foram avaliadas 40 mulheres, não tabagistas, com idades variando de 20 a 34 anos (média: 23,0; DP: 3,4). Quanto às profissões, $77,5 \%$ são estudantes, $2,5 \%$ são jornalistas, $15 \%$ fisioterapeutas, 2,5\% advogadas e $2,5 \%$ dentistas. Em relação à pratica de atividade física, $55 \%$ referem ser sedentárias, enquanto $45 \%$ praticam algum tipo de atividade física.

Em relação ao perfil das avaliadas, $65 \%$ não apresentava nenhum problema de visão diagnosticado, enquanto $35 \%$ relataram ter alguma alteração na visão. Quanto a problemas respiratórios, apenas 15\% possuíam alguma alteração respiratória diagnosticada. Das avaliadas, $40 \%$ relatavam apresentavam episódios constantes de dismenorreia. A frequência de constipa- 
Tabela 1 - Resultados das avaliações realizadas nos sujeitos do estudo.

\begin{tabular}{|c|c|c|c|}
\hline & Avaliação do multífido & $f$ & $\%$ \\
\hline (1.1) Avaliação de multífido das mulheres & Normal & 26 & 65,0 \\
\hline \multirow[t]{2}{*}{ nuligestas. } & Bom & 14 & 35,0 \\
\hline & $\begin{array}{l}\text { Variação da pressão na ativação da musculatura } \\
\text { profunda do abdome em } \mathrm{mmHg}\end{array}$ & $f$ & $\%$ \\
\hline (1.2) Variação da pressão na ativação & -15 & 1 & 2,5 \\
\hline da musculatura profunda do abdome em & -10 & 10 & 25,0 \\
\hline \multirow[t]{6}{*}{ mulheres nuligestas. } & -5 & 7 & 17,5 \\
\hline & 0 & 13 & 32,5 \\
\hline & 10 & 7 & 17,5 \\
\hline & 20 & 1 & 2,5 \\
\hline & 30 & 1 & 2,5 \\
\hline & Classificação da contração perineal & $\mathbf{f}$ & $\%$ \\
\hline \multirow{2}{*}{$\begin{array}{l}\text { (1.3) Classificação da força de contração } \\
\text { da musculatura perineal. }\end{array}$} & Normal & 31 & 77,5 \\
\hline & Insatisfatória & 9 & 22,5 \\
\hline
\end{tabular}

ção intestinal entre as avaliadas foi de $27,5 \%$.

$\mathrm{Na}$ avaliação do multífido, ${ }^{15} 65 \%$ das avaliadas possuíam função classificada como "normal", enquanto $35 \%$ das avaliadas foram classificadas como "bom" (Tabela 1 - 1.1).

No teste de ativação da musculatura profunda do abdome, a variação de pressão gerada variou de -15 a 30 $\mathrm{mmHg}$, sendo esta mantida por 10 segundos (Tabela 1 - 1.2).

Desta forma, a ativação do transverso do abdome foi classificada como insatisfatória em 55\% das mulheres. Quanto ao tempo de manutenção, este permaneceu na faixa da normalidade em todas as avaliadas.

$\mathrm{Na}$ avaliação das pressões respiratórias máximas com o manovacuômetro, a média da PImáx para as investigadas foi $98,25 \mathrm{cmH}_{2} \mathrm{O}$, enquanto a média da PEmáx foi $100,25 \mathrm{cmH}_{2} \mathrm{O}$. A classificação das pressões respiratórias máximas está descrita na figura 1 .

Os testes de estabilidade central buscaram avaliar a ativação muscular no complexo lombo-pélvico, pois os músculos profundos da coluna controlam a rigidez e a relação intervertebral. ${ }^{23} \mathrm{~A}$ instabilidade da coluna tem sido destacada como causa significativa de dor lombar e um fator causal em 20 a 30\% das doenças crônicas da coluna, neste estudo $75 \%$ das avaliadas apresentaram resultados abaixo do esperado para ativação da musculatura espinhal. ${ }^{24,25}$

A estabilidade da coluna é fornecida pelo funcionamento integrado dos sistemas de controle. ${ }^{10}$ Uma hipótese é que a função será restabelecida pelo recrutamento do sistema ativo. A fim de fazê-lo, Richardson e Jull $^{26}$ recomendam a ativação do transverso do abdome, multífido e do assoalho pélvico avaliados neste estudo que são músculos que têm baixos níveis de contração voluntária, durante os exercícios com carga. ${ }^{7}$

Observa-se que $65 \%$ das mulheres avaliadas mostraram ativar normalmente o multífido. Este músculo é responsável por manter a postura lordótica e realizar a extensão de tronco. Demonstra-se que a função do multífido está prejudicada em pacientes com sintoma de dor lombar. ${ }^{27,13}$ Os resultados do teste de abaixamento da perna estendida corroboram o estudo de Reinehr, ${ }^{2}$ realizado com mulheres com idade média de $23( \pm 1)$ anos, que mostraram um resultado classificados como deficitário neste teste aplicado anteriormente a um programa de treinamento de estabilização segmentar.

Entre as avaliadas, constatou-se que $55 \%$ não recrutam satisfatoriamente a musculatura profunda do abdome. Estudos demonstram a confiabilidade do uso de unidade de biofeedback pressórico para análise da ativação da musculatura profunda do abdome. ${ }^{28}$

Pesquisas mostram que o transverso abdominal é o principal músculo gerador da pressão intra-abdominal. ${ }^{4,29}$ Quando há ativação deste músculo, ocorre um aumento da pressão intra-abdominal, por conseguinte a estabilização da coluna é mantida. ${ }^{30} \mathrm{~A}$ ativação do transverso é independente da direção do movimento do

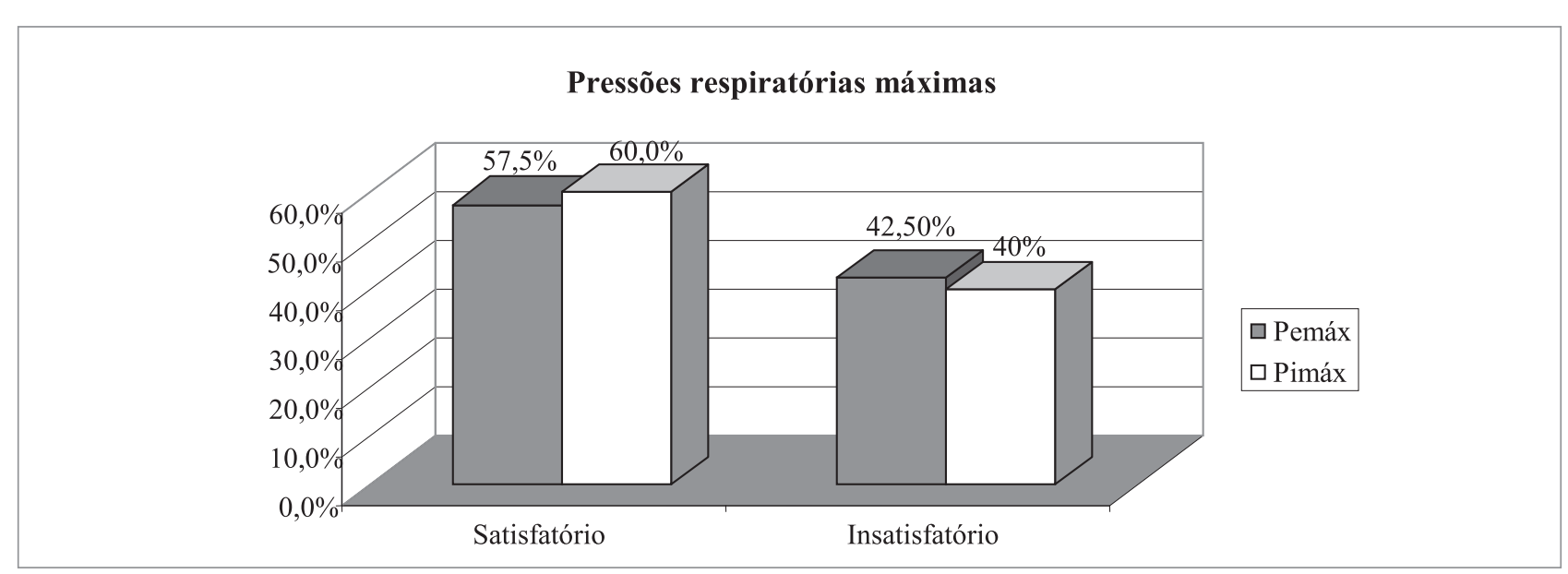

Figura 1 - Classificação das pressões respiratórias máximas em mulheres nuligestas. 
membro e é contínua durante todo o movimento dos membros inferiores. Estudo mostra que os músculos profundos do abdome apresentam atraso na ativação em pessoas com episódios recorrentes de dor lombar. ${ }^{4}$

$\mathrm{Na}$ avaliação da força da musculatura perineal, $77,5 \%$ das avaliadas obtiveram resultados satisfatórias, enquanto $22,5 \%$ apresentaram resultados abaixo do esperado. A musculatura do assoalho pélvico é ativada simultaneamente à ativação do transverso do abdome..$^{13}$ Uma contração efetiva da musculatura perineal ativa os músculos profundos do abdome, embora neste estudo não tenham sido encontrada correlação entre ativação da musculatura profunda do abdome e a força perineal das avaliadas. Estudo mostra que manter a musculatura abdominal relaxada durante a execução das contrações perineais pode afetar negativamente o desempenho desta contração. ${ }^{31}$

Além da função estabilizadora, os exercícios perineais possuem influência na sexualidade feminina. Em um estudo realizado com mulheres nuligestas constatou-se melhora na satisfação sexual, com aumento na frequência de orgasmos e maior desejo sexual, além do aumento na força perineal, após um protocolo de exercícios realizado em dez atendimentos. ${ }^{32}$

No presente estudo, não foi constatada a relação da força da musculatura perineal e a realização de atividade física, mas um estudo realizado com atletas evidenciou maior intensidade de contração no grupo não atletas, o que ressalta a importância da atividade física orientada, associando um trabalho direcionado aos músculos do assoalho pélvico. ${ }^{33}$

Nos resultados dos valores encontrados para Pimáx, $60 \%$ das investigadas obtiveram resultados satisfatórios, enquanto $40 \%$ alcançaram valores insatisfatórios. Na avaliação da Pemáx, 57,5\% apresentaram resultados satisfatórios, valores que discordam do estudo realizado com 103 indivíduos não fumantes, dos quais 56 eram mulheres, os valores para Pimáx foram significativamente menores do que os preditos de acordo com a equação de Neder et al. ${ }^{21} \mathrm{Um}$ outro estudo, ${ }^{34}$ realizado com 7 indivíduos, sendo 5 mulheres com idades entre 15 a 25 anos, demonstrou que estas obtiveram valores para Pimáx de em média - 83,5\% \pm $3,3 \%$ e para Pimáx em média $87, \% \pm 3,3 \%$.

Para executar eficientemente o ciclo respiratório é importante o equilíbrio da coluna, já que esta possibilitará a expansão necessária da caixa torácica. A respiração, portanto, afeta a estabilidade da coluna e, reciprocamente, a posição da coluna afeta a qualidade da respiração. ${ }^{35,36}$ A efetividade do diafragma depende da estabilidade da parede abdominal, que promove a sustentação das vísceras, durante a inspiração e depende também da estabilidade dos paravertebrais lombares, local da inserção vertebral do diafragma. Estes músculos impedem a elevação em bloco da caixa torácica. ${ }^{37,38}$

Toda alteração funcional provoca uma alteração em cadeia nos segmentos subjacentes, levando a adaptações em todo o complexo articular e muscular do corpo. Quando um músculo está deficiente há necessariamente uma sobrecarga em outro segmento corporal. Uma alteração em qualquer nível da coluna vertebral promove compensações nas curvaturas vizinhas. Dada à complexidade biomecânica da postura, é possível entender que, frente à alguma alteração muscular do complexo lombo-pélvico, ocorra refinamento dos sistemas de controle postural, sobrecargas das estruturas corporais próximas ou distantes através de compensações. Para que isso não ocorra é necessário o funcionamento harmônico dos músculos do centro de força. ${ }^{37}$

\section{CONSIDERAÇÕES FINAIS}

Este trabalho avaliou a ativação da musculatura do centro de força de mulheres nuligestas, trazendo informações quanto ao nível de estabilidade lombo-pélvico das avaliadas. Os sujeitos mostraram maior dificuldade na ativação dos músculos profundos do abdome, enquanto na ativação da musculatura do tronco, perineal e respiratória foram obtidos resultados satisfatórios.

É destacada a importância do uso da estabilidade muscular como conduta em diferentes atuações não somente na reabilitação, mas também na prevenção e promoção da saúde, uma vez que alterações foram encontradas.

\section{REFERÊNCIAS}

1. Ellenbecker TS. O joelho com problema. São Paulo: Manole; 2002.

2. Reinehr FB; Carpes FP; Mota CB. Influência do treinamento de estabilização central sobre a dor e a estabilidade lombar. Fis Mov 2008;21(1):123-129.

3. Kisner C, Colby LA. Exercícios terapêuticos: fundamentos e técnicas. 4.ed. São Paulo: Manole; 2005.

4. Hodges PW, Richardson CA. Inefficient muscular stabilization of the lumbar Spine associated with low back pain: a motor control evaluation of transverses abdominis. Spine 1996;21(22):2640-50.

5. Silva MC, Fassa AG, Valle NCJ. Dor lombar crônica em uma população adulta do sul do Brasil: prevalência e fatores associados. Cad Saúde Pub 2004;20(2):2115-22.

6. Matos MG, Hennington EA, Hoefel AL, Dias-Da-Costa JS. Dor lombarem usuários de um plano de saúde: prevalência e fatores associados. Cad Saúde Pub 2008;24(9):2115-22.

7. Mills JD, Taunton JE, Mills WA. The effect of a 10-week training regimen on lumbo-pelvic stability and athletic performance in female athletes: A randomized-controlled trial. Phys Ther in Sport 2005;(6)60-6.

8. Fritz JM, Erhard RE, Hagen BF. Segmental instability of the lumbar spine. Phys Ther 1998;(78):889-896.

9. Comeford MJ, Mottram SL. Movement and stability dysfunction -contemporary developments. Manual Ther 2001;6(1):15-26.

10. Panjabi, MM. The stabilizing system of the spine. Part I. Function, Dysfunction, Adaptation, and Enhancement. Journal of spin dis 1992;5(4):383-9.

11. Marshall PW, Murphy BA. Core stability exercises on and off a swiss ball. Arch Phys Med Rehabil 2005;96:242-9.

12. Goodman, PJ. Connecting the core. Perform Train Journal 2004;3(6):10-14.

13. Akuthota V, Nadler SF. Core Strengthening. Arch of Phys Med and Rehab 2004;85(85):86-92.

14. Lee D. A cintura pélvica. São Paulo: Manole; 2001.

15. Dutton M. Fisioterapia ortopédica. Porto Alegre: Artmed; 2006. 
16. Paciornick M. Aprenda a nascer e viver com os índios: parto de cócoras, desempenho sexual e ginástica indiana. Rio de Janeiro: Rosa dos tempos; 1997.

17. Moreno AL. Fisioterapia em Uroginecologia. São Paulo: Manole; 2004.

18. Barboza AMP, Carvalho LR, Martins AMVC, Calderon IMP, Rudge MVC. Efeito da via parto sobre a força muscular do assoalho pélvico. Rev Bras Ginecol Obstet 2005;27(11):677-682.

19. Amaro JL, Haddad JM, Trindade JCS, Ribeiro RM. Reabilitação do assoalho pélvico nas disfunções urinárias e anorretais. São Paulo: Segmento Farma; 2005.

20. Rocha JMS. Ganho de força muscular respiratória com uso de inspirómetro incentivador. Rev dig vida saúde, 2002 out-nov, [cited 2009 nov 20]. Disponível em: http://www. revistadigitalvidaesaude.hpg.com.br/artv1n2 _ 09.pdf.

21. Pereira VF, França DC, Zampa CC, Fonseca MM, Tomich GM, Britto RR. Pressões respiratórias máximas : Valores encontrados e preditos em indivíduos saudáveis. Rev Bras Fis 2007;11(5):361-8.

22. Neder JA, Andreoni S, Lerario MC, Nery LE. Reference values for lung function tests. II. Maximal respiratory pressures and voluntary ventilation. Braz J Med Biol Res 1999;32(6):719-27.

23. Oliveira VC, Bicalho IL, Soares TB, Dornellas RS. Estabilidade articular da coluna vertebral: teorias contemporâneas e novos paradigmas.Fis Brasil 2009;10(4):284-9.

24. Apperley S. Differential functioning of deep and superficial lumbar multifidus fibres during vertebral indentation perturbations [thesis]. Vancouver: University of British Columbia; 2008.

25. Watson C. The effects of pilates training on the function of thetransversus abdominis [dissertation]. Washington: Faculty of Western Washington University, 2006.

26. Hides JA, Richarson CA, Jull GA. Multifidus muscle recovery is not automatic after resolution of acute, first-episode low back pain. Spine 1996;21(23):2763-2769.

27. Kolyniak IEGG, Cavalcanti SMB, Aoki MS. Avaliação isocinética da musculatura envolvida na flexão e extensão do tronco: efeito do método Pilates. Rev Bras Med Esp 2004;10(6):487-90.
28. Costa LOP, Costa LCM, Cançado RL, Oliveira WM, Ferreira $\mathrm{PH}$. Confiabilidade do teste palpatório e da unidade de biofeedback pressórico na ativação do músculo transverso abdominal em indivíduos normais. Acta Fisiatr 2004;11(3):101-105.

29. Gouveia KMC, Gouveia EC. O músculo transverso abdominal e sua função de estabilização da coluna lombar. Fisioter. Mov 2008;21(3):45-50.

30. Cynn HS, Seop J, Kwon OY, Yi CH.Effects of lumbar stabilization using a pressure biofeedback unit on muscle activity and lateral pelvic tilt during hip abduction in sidelying. Arch Phys Med Rehabil 2006;87:1454-8.

31. Neuman P, Gill V. Pelvic Floor and Abdominal Muscle Interaction: EMG Activity and Intra-abdominal Pressure. Int Urogynecol J 2002;13:125-132.

32. Bianco G, Braz MM. Efeitos dos exercícios do assoalho pélvico na sexualidade feminina [monografia]. Tubarão: Universidade do Sul de Santa Catarina; 2004.

33. Larosa AV, Magnani OS, Oliveira, J, Mesquita RA. Comparação da funcionalidade do assoalho pélvico entre mulheres atletas e não-atletas [monografia]. Araraquara: Centro Universitário de Araraquara.

34. Batista JBS, Lima MC. O iso-stretching no ganho de expansibilidade torácica e força muscular respiratória em indivíduos saudáveis. II Seminário de Fisioterapia da UNIAMERICA: Iniciação Científica, 5 e 6 de maio 2008, Foz do Iguaçu.

35. Ferreira LR, Banov AD, Bankoff $P$. Influência da respiração na melhora da postura corporal em praticantes da dança: um estudo de caso. Mov \& Percep. 2009;10(14):1679-8678.

36. Hodges PW, Butler JE, McKenzie DK, Gandevia SC. Contraction of the human diaphragm during rapid postural adjustments. Journal of Physiology 1997;505(2):539-548.

37. Yi LC, Jardim JR, Inoue DP, Pignatari SNS. Relação entre a excursão do músculo diafragma e as curvaturas da coluna vertebral em crianças respiradoras bucais. J. Pediatr 2008;84(2):171-177.

38. Benatti AT. Equilíbrio tóraco-abdominal: ação integrada à respiração e à postura. Arq ciênc. Saúde unipar 2001;5(1):87-92.

Como citar: FRIGO, Letícia Fernandez; BRAZ, Melissa Medeiros. Avaliação da ativação muscular do centro de força de mulheres nuligestas. Cinergis, Santa Cruz do Sul, v. 17, n. 2, jun. 2016. ISSN 2177-4005. Disponível em: < https://online. unisc.br/seer/index.php/cinergis/article/view/6737>. Acesso em: 04 jul. 2016. doi:http://dx.doi.org/10.17058/cinergis. v17i2.6737. 